\title{
Metformina en el tratamiento de la diabetes tipo 2 con sobrepeso u obesidad
}

\author{
M. C. MARTÍN MUÑOZ, J. M. DÍAZ, J. M. MUROS BAYO, A. GONZÁLEZ ÁLVARO, \\ P. COSTA ZAMORA
}

Centro de Salud Calesas y Hospital Universitario 12 de Octubre (Área 11). Madrid

METFORMIN IN THE TREATMENT OF TYPE 2 DIABETES IN OVERWEIGTHED OR OBESE PATIENTS

\section{RESUMEN}

Fundamento y objetivos: La Diabetes es la enfermedad crónica cuya prevalencia ha sufrido un mayor incremento en los últimos años. La obesidad es el factor de riesgo más importante para presentar esta enfermedad y la metformina se considera una terapia de primera línea en diabéticos obesos. El objetivo de este estudio es conocer la utilización de metformina y evaluar el grado de control de los factores de riesgo cardiovascular (FRC) asociados, en los pacientes con DM tipo 2 en situación de sobrepeso u obesidad.

Pacientes y método: Estudio descriptivo trasversal sobre una muestra aleatoria de 308 pacientes en las consultas de atención primaria. Se clasificaron los sujetos según el tratamiento antidiabético recibido en cuatro grupos (dieta, insulina, metformina y otros antidiabéticos orales). Se evaluaron el grado de control de los FRC modificables, la existencia de complicaciones y el control metabólico de la población estudiada.

Resultados: La edad media fue de 69,6 años, el 54\% eran mujeres. El $36,6 \%$ de los pacientes seguían tratamiento con metformina y el $40,5 \%$ con sulfonilureas. La afectación macrovascular fue del 19,4\%. El FRC más frecuente resultó la hipertensión arterial en el 76\%. Los objetivos de control menos alcanzados fueron el cLDL $<100 \mathrm{mg} / \mathrm{dl}$ y TAS $<130$ $\mathrm{mmHg}$. Los diabéticos tratados sólo con dieta tuvieron mejores controles de glucemia basal y $\mathrm{HbA} 1 \mathrm{c} \%$ que los tratados con metformina $(\mathrm{p}<$ $0,001)$ e insulina $(\mathrm{p}<0,001)$. El grupo tratado con metformina presentó valores de triglicéridos (TG) significativamente más elevados que el grupo de dieta $(\mathrm{p}=0,009)$ e insulina $(\mathrm{p}=0,041)$.

Conclusiones: A pesar de que el tratamiento indicado es la metformina, ésta se utiliza en una proporción similar a las sulfonilureas en DM tipo 2 con sobrepeso u obesidad. Existe un pobre control de la mayoría de los FRC de la población diabética estudiada.

PALABRAS CLAVE: Diabetes mellitus tipo 2. Control glucémico. Metformina. Obesidad.

\section{ABSTRACT}

Fundament and objectives: Diabetes is the disease that has suffered a greater increase in his incidence in the last years. Obesity is the most important risk factor to develop this disease and metformin is considered as a first line drug in overweighted diabetic patients. The aim of this study is to evaluate the use of metformin and the degree of control of the associated cardiovascular risk factors (CVRF) in patients suffering of type 2 diabetes and overweight or obesity.

Patients and methods: This is a transversal descriptive study over a random chosen group of 308 patients followed at primary care level. They were classified in four different groups depending on the treatment used to control them (diet, insulin, metformin and other oral antidiabetic drugs). They were evaluated upon the degree of control of the modifiable $C V R F$, the presence of complications and the metabolic control.

Results: The average age was 69,6 years, 54\% of the patients female. $36,6 \%$ followed treatment with metformin and 40,5\% with sulfonylureas. The macrovascular percentage of affection was 19,4\%. The most frequent CVRF was arterial hypertension (76\%). The goal levels of control that were less in range were the levels of $c L D L<100 \mathrm{mg} / \mathrm{dl}$ and systolic blood pressure $<130 \mathrm{mmHg}$. Diabetic patients only treated with diet had a better control of fasten glycemia and HbAlc\% than those ones treated with metformin $(p<0.001)$. The group treated with metformin showed levels of triglycerides (TG) significantly higher than those of the diet controlled group $(p=0.009)$ and insulin group $(p<0.001)$.

Conclusions: Metformin and sulfonylureas are used in a similar proportion in type 2 diabetics with overweight or obesity. There is a poor control of the majority of the CVRF in the diabetic population studied.

KEY WORDS: Diabetes mellitus type 2. Metabolic control. Metformin. Obesity.

Martín Muñoz, MC, Martín Díaz, J, Muros Bayo JM, González, Álvaro A, Costa Zamora P. Metformina en el tratamiento de la diabetes tipo 2 con sobrepeso u obesidad. An Med Interna (Madrid) 2005; 22: 579-585.

\section{INTRODUCCIÓN}

La diabetes mellitus (DM) es una enfermedad metabólica crónica y compleja que se caracteriza por deficiencia absoluta o relativa de insulina, hiperglucemia crónica y otras alteracio- nes del metabolismo de los carbohidratos y de los lípidos; ello a su vez puede originar múltiples complicaciones microvasculares en los ojos, el riñón y las extremidades inferiores, así como neuropatías periféricas $\mathrm{y}$, frecuntemente, lesiones macrovasculares y coronarias1. Los pacientes con DM tipo 2

Trabajo aceptado: 29 de julio de 2005 
tienen un riesgo de cardiopatía isquémica $(\mathrm{CI})$ equivalente al de personas no diabéticas que han sufrido un episodio previo (2). La asociación de obesidad abdominal, hipertensión arterial, disminución de la tolerancia a la glucosa y dislipemia conlleva un aumento del riesgo cardiovascular y constituye el llamado síndrome plurimetabólico. El origen de este síndrome es probablemente la presencia de una resistencia a la insulina en el tejido adiposo, hepático y muscular. Existen evidencias en la literatura que demuestran que la corrección de los diferentes FRC modificables se asocia a una disminución de la incidencia de eventos cardiovasculares en los pacientes con DM tipo 2 (3).

Es la enfermedad crónica cuya prevalencia ha sufrido un mayor incremento en los últimos años (4). La prevalencia global de diabetes en los países desarrollados se estima en un 3-6 $\%$ de la población general (16\% en mayores de 65 años) con una clara tendencia a aumentar, debido al envejecimiento de la población, al sedentarismo y a la mayor prevalencia de la obesidad (5). La obesidad es el factor de riesgo más importante para presentar DM tipo 2. La frecuencia de presentación de diabetes en la población obesa es 2,9 veces superior a la no obesa y un $80 \%$ de los diabéticos tipo 2 tiene un índice de masa corporal (IMC) mayor a 27 en el momento del diagnóstico. En el riesgo de diabetes influyen también: la duración de la obesidad y las variaciones del peso. Incrementos de peso, aunque sean pequeños, se relacionan con un aumento del riesgo de desarrollar diabetes.

La verdadera importancia de la diabetes como problema de salud está relacionada con el desarrollo de complicaciones macro y microvasculares, que determinan el curso clínico y pronóstico de la enfermedad, produciendo tasas muy elevadas de invalidez y muerte (6). Por otro lado es un problema de salud al que se le dedican importantes recursos económicos en la actualidad.

La importancia del control glucémico en la prevención de las complicaciones microvasculares se ha confirmado en pacientes con diabetes tipo1 y tipo 27. La principal causa de mortalidad en la DM tipo 2 es cardiovascular (8). Para reducir la morbimortalidad por esta causa se han mostrado eficaces el control lipídico y el control de la tensión arterial (9), mientras que la eficacia del control glucémico es más controvertida (3). Por ello, las sociedades de diabetes europea (10) y americana (11) han recomendado la consecución de unos objetivos de control glucémico, lipídico y de tensión arterial cada vez más estrictos, con el objetivo de reducir las complicaciones crónicas micro y macroangiopáticas del paciente diabético.

El programa de atención a las personas con DM, se encuentra entre los más consolidados en la práctica de los profesionales de Atención Primaria. Los avances científicos producidos en los últimos años en el estudio de la diabetes han obligado a actualizar las propuestas de actuación, buscando un abordaje más integral de la enfermedad y un control más efectivo del conjunto de los FRC (1).

Aunque el tratamiento inicial de la DM tipo 2 incluye medidas higienicodietéticas, la mayoría de los pacientes requieren intervención farmacológica para mantener unos controles glucémicos adecuados. Los pacientes obesos, además muestran una resistencia insulínica asociada al déficit relativo de insulina y en algunos casos requirieren dosis muy elevadas de ésta para controlar la hiperglucemia (12). Existen problemas relacionados con la alta dosis de insulina (13). Estudios en animales y datos epidemiológicos sugieren que puede haber una relación entre la hiperinsulinemia y la formación de ateroesclerosis y enfermedad cardiovascular. Tanto la terapia con sulfonilureas como la insulina se han asociado a efectos indeseables clínicamente significativos: elevados niveles de insulina en plasma, aumento del peso corporal y existe un mayor riesgo de hipoglucemias (13).

La metformina, un derivado biguanídico clasificado como antihiperglucémico, no está asociado a hiperglucemia significativa o hiperinsulinemia periférica3. Se ha descrito que la monoterapia con metformina reduce los niveles de triglicéridos y de colesterol LDL en diabéticos, obesos o no (13). Sus efectos favorables en el peso corporal y en los lípidos del plasma sugieren a los científicos considerar la metformina como una terapia de primera línea en diabéticos obesos y puede utilizarse la terapia combinada de metformina más sulfonilureas en pacientes con DM tipo 2 obesos (3). La acción antihiperglucémica de la metformina viene de su capacidad para inhibir la producción de glucosa hepática y en menor grado de la absorción y utilización de la glucosa periférica por las células musculares principalmente (14). Otros mecanismos que contribuyen son, el incremento de utilización de glucosa por el intestino y la reducida tasa de oxidación de los ácidos grasos (13).

Durante muchos años la metformina ha arrastrado la sombra del peligro de la acidosis láctica. El estudio UKPDS muestra que en pacientes con DM tipo 2 obesos, la elección de metformina como fármaco de primera línea ante el fracaso del tratamiento dietético conlleva mayores beneficios que riesgos, cuando se compara con el tratamiento con otros fármacos (sulfonilureas o insulina) (3). Reduce el riesgo no sólo de complicaciones microvasculares, sino también de las macrovasculares y la mortalidad. Este estudio es la primera prueba que demuestra una reducción del riesgo de la enfermedad cardiovascular en el tratamiento farmacológico del paciente con DM tipo 2.

El propósito de este trabajo ha sido conocer la utilización de metformina, y evaluar el grado de control de los FRC asociados, en los pacientes con DM tipo 2 en situación de sobrepeso u obesidad. La muestra ha sido seleccionada de las consultas de Atención Primaria.

\section{MATERIAL Y MÉTODOS}

Diseño: Se trata de un estudio descriptivo transversal, consiste en la revisión de las historias clínicas de los pacientes.

Población a estudio: De una población de 28.888 pacientes en el centro de salud de Calesas (Área 11 de Madrid), 732 con registro de DM tipo 2 e IMC mayor de 25 , se seleccionó de forma aleatoria una muestra de 401 pacientes en las consultas de Atención Primaria. De la muestra seleccionada, 308 pacientes cumplieron los criterios de inclusión.

Los criterios de inclusión fueron:

1. Diabetes tipo 2 diagnosticada según los criterios de la ADA (17)

2. IMC mayor o igual de 25

3. Edad mayor a 18 años

4. Registro de al menos una visita en la historia clínica, desde el 1 de junio al 31 de diciembre de 2003.

Los criterios de exclusión fueron:

1. Tiempo de evolución de la diabetes menor de 6 meses

2. sensibilidad a la metformina 
3. Paciente terminal o crónico en visita a domicilio.

4. insuficiencia hepática crónica, alcoholismo y otras toxicodependencias.

5. insuficiencia renal crónica en diálisis.

6. insuficiencia cardiaca crónica refractaria, infarto agudo de miocardio (IAM) en los 3 meses previos al estudio o cardiopatía isquemica inestable.

7. Demencia y enfermedades psiquiátricas graves con deterioro cognitivo.

Variables analizadas. Los datos se recogieron según protocolo estandar a partir de las historias clínicas.

Se incluyeron: la edad y el sexo.

Variables clínicas: peso, talla, IMC, tensión arterial sistólica (TAS) y diastólica (TAD)

Variables bioquímicas: glucemia, hemoglobina glicada (Hg A1c), colesterol total, colesterol HDL y LDL, triglicéridos, creatinina y microalbuminuria.

Datos de afectación orgánica por DM a nivel microvascular (retinopatía diabética, nefropatía y/o neuropatía) y macrovascular (cardiopatía isquémica, enfermedad cerebrovascular isquémica y/o enfermedad vascular periférica) y datos sobre FRC asociados (tabaquismo actual, hipertensión arterial y/o hipercolesterolemia).

Variables de tratamiento: antidiabéticos (sulfonilureas, biguanidas, secretagogos de acción rápida, glitazonas, inhibidores alfa glucosidasas o insulina), antihipertensivos (diuréticos, betabloqueantes, IECA, ARA-II, antagonistas del calcio, bloqueantes alfaadrenérgicos) y uso de hipolipemiantes y/o antiagregantes.

Los pacientes se clasificaron en cuatro grupos según el tratamiento antidiabético recibido: dieta únicamente, metformina sin insulina con o sin otros antidiabéticos orales, otros antidiabéticos orales sin insulina, e insulina con o sin otros antidiabéticos orales.

Para evaluar el grado de control de los FRC modificables de la población estudiada, nuestros resultados se compararon con los objetivos de control de las recomendaciones de la ADA.

Métodos estadísticos: se calculó el tamaño muestral para un nivel de confianza (1-alfa) de 0,95 una precisión de +/0,05 y una proporción esperada de pacientes tratados con metformina del $50 \%$. Considerando las posibles exclusiones se aumentó el tamaño de la muestra un 5\%.

Los datos se analizaron mediante el programa estadístico SPSS versión 11.0. La estadística descriptiva utilizada para las variables cuantitativas se basó en el calculo de medias y desviaciones estándar y para las variables cualitativas en el cálculo de proporciones.

Se aplicó la prueba de Kolmogorov-Smirnov para comprobar la normalidad de las variables.

Las pruebas de estadística analítica utilizadas para la comparación de proporciones fueron el test de la ji cuadrado o en el caso de no cumplir las condiciones necesarias para su aplicación la prueba exacta de Fisher. Para la comparación de dos medias se utilizó la t de student o la U de Mann-Whytney en el caso de no cumplir supuestos de normalidad. Para la comparación de más de dos medias se utilizó el análisis de la varianza (ANOVA) o el test de KruskalWallis y el test de Scheffé para determinar entre que medias había diferencias.

En todas las pruebas se consideró estadísticamente significativo los valores de $\mathrm{p}<0,05$.

\section{RESULTADOS}

El número de individuos estudiados a partir de historias clínicas informatizadas susceptibles de evaluación fueron 308 pacientes. De ellos 167 (54\%) eran mujeres. La edad media fue de 69,6 años (DE 10,1). La descripción de las variables analizadas queda reflejada en las tablas I y II.

Había $102(33 \%)$ pacientes tratados con dieta, 125 $(40,5 \%)$ con sulfonilureas, $113(36,6 \%)$ con metformina y 39 $(12,6 \%)$ con insulina, combinaron sulfonilureas con metformina 59 (19\%) e insulina con metformina 16 (5,2\%). Los porcentajes de tratamientos hipoglucemiantes se reflejan en la figura 1 .

TABLA I

DESCRIPCIÓN DE LOS PORCENTAJES DE LAS VARIABLES DEL ESTUDIO $n=309$

\begin{tabular}{lrrc}
\hline & $n$ & $\%$ & $\begin{array}{c}\text { Intervalo de } \\
\text { confianza del 95\% }\end{array}$ \\
\hline $\begin{array}{lrr}\text { Sexo (mujeres) } \\
\text { (varones) }\end{array}$ & 167 & 54 & $48,3-59,7$ \\
$\begin{array}{l}\text { Complicaciones } \\
\text { microvasculares }\end{array}$ & 142 & 46 & $40,3-51,7$ \\
Complicaciones & 30 & 9,7 & $6,3-13$ \\
macrovasculares & 60 & 19,4 & $14,9-23,9$ \\
Hipertensión arterial & 235 & 76 & $71,1-80,8$ \\
Hipercolesterolemia & 160 & 51,7 & $46-57,4$ \\
Tabaquismo & 42 & 13,6 & $9,7-17,5$ \\
\hline
\end{tabular}

TABLA II

DESCRIPCIÓN DE LAS MEDIAS DE LAS VARIABLES DEL ESTUDIO $\mathrm{n}=309$

\begin{tabular}{lrr}
\hline & Media; DE & \multicolumn{1}{c}{$\begin{array}{c}\text { Intervalo de } \\
\text { confianza del } 95 \%\end{array}$} \\
\hline Edad, años & 69,$6 ; 10,2$ & $68,5-70,7$ \\
IMC (Kg/ talla m²) & 30,$9 ; 4,2$ & $30,4-31,4$ \\
TAS (mmHg) & 138,$2 ; 15,2$ & $136,5-140$ \\
TAD (mmHg) & 78,$2 ; 9,5$ & $77,1-79,3$ \\
Colesterol (mg/dl) & 201,$3 ; 35,3$ & $196,7-205,8$ \\
Triglicéridos (mg/dl) & 147,$9 ; 93,4$ & $135,7-160,1$ \\
c-HDL (mg/dl) & 52,$8 ; 14,2$ & $50,9-54,8$ \\
c-LDL (mg/dl) & 119,$6 ; 30,3$ & $115,4-123,8$ \\
HbA1c (\%) & 6,$5 ; 1,4$ & $6,3-6,7$ \\
Glucemia basal (mg/dl) & 146,$3 ; 43,5$ & $140,8-151,8$ \\
Microalbuminuria (mg/L) & 26,$7 ; 117,7$ & $7,9-45,5$ \\
Creatinina (mg/dl) & 0,$88 ; 0,37$ & $0,84-0,93$
\end{tabular}

IMC: indice de masa corporal. TAS: tensión arterial sistólica. TAD: tensión arterial diastólica.

c-HDL: colesterol unido a lipoproteínas de alta densidad.

c-LDL: colesterol unido a lipoproteínas de baja densidad

La comparación de las variables en los distintos grupos de tratamiento en los que fueron clasificados los pacientes se refleja en la tabla III. 


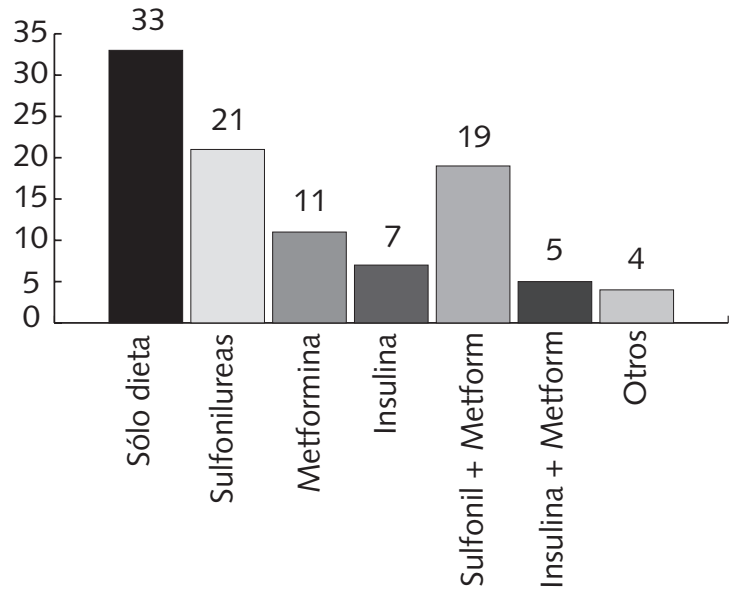

Fig. 1. Tratamientos hipoglucemiantes utilizados.

Los pacientes tratados sólo con dieta tenían más edad $(71,8 \pm 10,5)$ que el grupo tratado con metformina $(67,7 \pm$ $10,5) \mathrm{p}=0,042$. La media de la glucemia basal (GB) para todos los pacientes fue de $146,3 \pm 43,5 \mathrm{mg} / \mathrm{dL}$, en el grupo de pacientes con sólo dieta fue significativamente menor que en el resto de grupos $(\mathrm{p}<0,001)$. El valor medio de la $\mathrm{Hb}$ Aic fue de 6,5 $\pm 1,4$, los pacientes tratados sólo con dieta (HbA1c 5,8 \pm 1 ) presentaron unos niveles significativamente menores a los tratados con metformina (HbA1c 6,9 $\pm 1,3) \mathrm{p}<0,001 \mathrm{e}$ insulina (HbA1c 7,4 $\pm 1,4) \mathrm{p}<0,001$. En el grupo de otros ADOS (HbA1c 6,4 $\pm 1,4)$ los valores fueron significativamente menores al grupo de insulina $\mathrm{p}=0,013$.

La media de los triglicéridos en todos los pacientes fue de $147,9 \pm 93,4 \mathrm{mg} / \mathrm{dl}$, el grupo de metformina $(183,6 \pm 141,6)$ tuvo significativamente valores más elevados que en los grupos de dieta $(131,4 \pm 60,1) \mathrm{p}=0,009$ e insulina $(124,7 \pm 50,6)$ $p=0,041$. No hubo diferencias estadísticas significativas entre los grupos en relación al sexo, TAS, TAD, colesterol, colesterol-HDL, colesterol-LDL, creatinina, FRC y complicaciones macrovasculares. No se observaron complicaciones microvasculares en el grupo de sólo dieta y fueron significativamente más frecuentes en el grupo de insulina respecto a los demás grupos $p<0,000$. No se encontraron diferencias significativas entre los valores de $\mathrm{HbA} 1 \mathrm{c}$ por sexo y grupos de edad.

El número de pacientes con objetivos de control alcanzados en toda la muestra se reflejan en la tabla IV y el análisis de objetivos alcanzados por grupos de tratamiento en la tabla V. De acuerdo con los criterios de control definidos, los objetivos menos alcanzados fueron: colesterol-LDL $<100 \mathrm{mg} / \mathrm{dl}$ en el $16,2 \%$, TAS $<130 \mathrm{mmHg}$ en el $17,1 \%$ y glucemia basal $<120 \mathrm{mg} / \mathrm{dl}$ en el $19,4 \%$, y los más alcanzados fueron: no fumar en el 86,4\%, colesterol-HDL > $40 \mathrm{mg} / \mathrm{dl}$ en el 56,3\% y $\mathrm{TG}<150 \mathrm{mg} / \mathrm{dl}$ en el $50,5 \%$. Una TA $<130 / 80$ se observó en 38 pacientes $(12,3 \%)$. Únicamente se logró el control de todos los objetivos en 4 pacientes $(1,3 \%)$.

TABLA III

COMPARACIÓN DE LAS VARIABLES POR GRUPOS DE TRATAMIENTO

\begin{tabular}{|c|c|c|c|c|c|}
\hline & $\begin{array}{c}\text { Dieta } \\
n=102\end{array}$ & $\begin{array}{c}\text { Metformina } \\
n=97\end{array}$ & $\begin{array}{c}\text { Otros ADOs } \\
n=71\end{array}$ & $\begin{array}{c}\text { Insulina } \\
n=39\end{array}$ & Significación \\
\hline Edad (años) & $71,8 \pm 10,5$ & $67,7 \pm 10,5$ & $70,2 \pm 9,1$ & $67,3 \pm 8,8$ & $p<0.013$ \\
\hline Varones & $44,1 \%$ & $49,5 \%$ & $50,7 \%$ & $33,3 \%$ & NS \\
\hline Mujeres & $55,9 \%$ & $50,5 \%$ & $49,3 \%$ & $66,7 \%$ & NS \\
\hline IMC (Kg/talla m²) & $30,6 \pm 3,8$ & $31,6 \pm 5,0$ & $30,6 \pm 3,7$ & $30,8 \pm 4,1$ & NS \\
\hline TAS $(\mathrm{mmHg})$ & $138,7 \pm 14,8$ & $136,6 \pm 14,4$ & $138,4 \pm 16,7$ & $141,1 \pm 15,7$ & NS \\
\hline TAD (mmHg) & $77,6 \pm 10,4$ & $78,2 \pm 9,0$ & $79,2 \pm 8,3$ & $78,3 \pm 9,8$ & NS \\
\hline Colesterol total $(\mathrm{mg} / \mathrm{dl})$ & $201,0 \pm 36,9$ & $206,4 \pm 37,8$ & $199,2 \pm 33,4$ & $194,4 \pm 31,0$ & NS \\
\hline Triglicéridos (mg/dl) & $131,4 \pm 60,1$ & $183,6 \pm 141,6$ & $142,1 \pm 62,7$ & $124,7 \pm 50,6$ & $p<0.002$ \\
\hline $\mathrm{cHDL}(\mathrm{mg} / \mathrm{dl})$ & $53,6 \pm 13,4$ & $53,0 \pm 15,1$ & $50,4 \pm 12,2$ & $54,5 \pm 17,1$ & NS \\
\hline $\mathrm{cLDL}(\mathrm{mg} / \mathrm{dl})$ & $121,1 \pm 30,5$ & $119,4 \pm 31,7$ & $118,8 \pm 29,3$ & $117,8 \pm 29,5$ & NS \\
\hline $\mathrm{HbA1c}(\%)$ & $5,8 \pm 1,0$ & $6,9 \pm 1,3$ & $6,4 \pm 1,4$ & $7,4 \pm 1,4$ & $p<0.001$ \\
\hline Glucemia basal (mg/dl) & $129,6 \pm 23,8$ & $156,9 \pm 38,0$ & $151,3 \pm 56,8$ & $158,2 \pm 55,6$ & $p<0.001$ \\
\hline Microalbuminuria (mg/l) & $7,2 \pm 14,8$ & $11,5 \pm 39,1$ & $23,9 \pm 41,8$ & $104,9 \pm 285,7$ & NS \\
\hline Creatinina $(\mathrm{mg} / \mathrm{dl})$ & $0,9 \pm 0,2$ & $0,9 \pm 0,6)$ & $0,8 \pm 0,2$ & $0,8 \pm 0,2$ & NS \\
\hline \multicolumn{6}{|l|}{ Hipertensión } \\
\hline arterial & $80,4 \%$ & $75,3 \%$ & $70,4 \%$ & $76,9 \%$ & NS \\
\hline Dislipemia & $46,1 \%$ & $59,8 \%$ & $43,7 \%$ & $61,5 \%$ & NS \\
\hline Tabaquismo & $14,7 \%$ & $14,9 \%$ & $11,3 \%$ & $10,3 \%$ & NS \\
\hline \multicolumn{6}{|l|}{ Complicación } \\
\hline microvascular & $0,0 \%$ & $12,4 \%$ & $4,2 \%$ & $38,5 \%$ & $p<0.001$ \\
\hline \multicolumn{6}{|l|}{ Complicación } \\
\hline macrovascular & $13,7 \%$ & $21,6 \%$ & $21,1 \%$ & $25,6 \%$ & NS \\
\hline
\end{tabular}

Otros ADOs incluyen cualquier antidiabético oral excepto la metformina.

Los resultados expresan la media \pm desviación estándar o el pocentaje

IMC: indice de masa corporal. TAS: tensión arterial sistólica. TAD: tensión arterial diastólica. c-HDL: colesterol unido a lipoproteínas de alta densidad.

c-LDL: colesterol unido a lipoproteínas de baja densidad 
Comparando los 4 grupos se observa que el porcentaje de pacientes tratados sólo con dieta que alcanzaron el objetivo de control de $\mathrm{HbA} 1 \mathrm{c} \%<7 \%$ fue significativamente superior al resto de grupos $\mathrm{p}<0,001$, y el porcentaje de pacientes del grupo de metformina que alcanzó el objetivo de control de glucemia basal $<120 \mathrm{mg} / \mathrm{dl}$ y $\mathrm{TG}<150 \mathrm{mg} / \mathrm{dl}$ fue significativamente inferior al resto de grupos, $\mathrm{p}=0,049 \mathrm{y} \mathrm{p}=0,043$ respectivamente.

Al comparar los objetivos de control lipídico en relación a la existencia o no de tratamiento hipolipemiante no se encontraron diferencias significativas. Sí existe de forma significativa mejor control de la TAS en los pacientes que utilizan fármacos antihipertensivos frente a los que no los utilizan $\mathrm{p}=$ 0,001. El FRC más frecuente resultó la hipertensión (HTA) en el $76 \%$ de esta muestra, seguido de hipercolesterolemia en el $51,7 \%$ y tabaquismo en el $13,6 \%$. Presentaron más de uno de estos FRC el 44\%. No hubo diferencias significativas entre el grado de control de la $\mathrm{HbA} 1 \mathrm{c} \%$ y la presencia de FRC.

Respecto a complicaciones propias de esta enfermedad, la más frecuente fue la afectación macrovascular en un 19,4\% (CI en el $11,7 \%$ y accidente cerebrovascular (ACVA) en el $6,8 \%$ ), la complicación microvascular se detectó en el 9,7\%. Un mejor control de la $\mathrm{HbA} 1 \mathrm{c} \%$ estaba relacionado con significación límite a la no afectación microvascular $\mathrm{p}=0,046$.

El 77,6\% están en tratamiento con antihipertensivos, siendo los más utilizados los diuréticos $(42,7 \%)$ y los IECA (40,8\%), con hipolipemiantes el 43,7\% y con antiagregantes y/o anticoagulantes el 34,6\%. El tratamiento con ARA 2 se utiliza significativamente en mayor proporción en el grupo de insulina y en menor proporción en el grupo de dieta $\mathrm{p}<0,008$. El tratamiento hipolipemiante se utiliza significativamente más en el grupo de insulina y en el grupo de metformina y menos en el grupo de dieta $\mathrm{p}<0,017$. El tratamiento antiagregante se utiliza significativamente en mayor proporción en el grupo de insulina y en menor proporción en el grupo de dieta $\mathrm{p}<0,007$. No hay diferencias significativas en la utilización de los fármacos diuréticos, IECAS, betabloqueantes o antagonistas del calcio entre los cuatro grupos.

\section{DISCUSIÓN}

La asociación entre hiperglucemia y riesgo de complicaciones micro y macrovasculares de la diabetes ha suscitado considerable interés científico. Hay gran cantidad de referencias bibliográficas que indican que hay una relación directa entre la magnitud de hiperglucemia y el riesgo y progresión de estas complicaciones. La naturaleza progresiva de la diabetes tipo 2 como resultado de la disminución de la función secretora de insulina del páncreas y la resistencia a la insulina representa un reto para el logro del mantenimiento de la normoglucemia. Estudios a largo plazo apoyan la observación clínica de que el control glucémico se pierde progresivamente con cualquier agente oral simple. Se requieren múltiples terapias para mantener la normoglucemia (15). En nuestro estudio existe significación estadística entre el mejor control glucémico $(\mathrm{HbA} 1 \mathrm{c} \%)$ y la menor afectación de complicaciones microvasculares. No existiendo diferencias significativas respecto a las complicaciones macrovasculares.

El estudio UKPDS evaluó los efectos del control de glucosa en sangre con metformina sobre el riesgo y progreso de las complicaciones clínicas en pacientes obesos con DM tipo 2 tratados con dieta (3). En pacientes obesos con DM tipo 2 en tratamiento con metformina se reducen las complicaciones macro y microvasculares de la diabetes y todas la causas de mortalidad, en relación con una política de control glucémico con sulfonilureas e insulina. Además produce una menor ganancia ponderal y una menor incidencia de efectos secundarios que los pacientes tratados con sulfonilureas e insulina. Aunque el ensayo se realizó en ámbito hospitalario, en Atención Primaria podrían obtenerse similares resultados. Los pacientes incluidos en el UKPDS son diabéticos tipo 2 de nuevo diagnóstico, no controlados con tratamiento dietético, con características similares a los que atendemos en las consultas de Atención Primaria.

La metformina se considera una terapia de primera línea en diabéticos obesos. Además se asocia a la mejora de los parámetros lipídicos en pacientes con y sin DM, independientemente de sus efectos hipoglucémicos. Los efectos colaterales más frecuentes asociados a terapia con metformina son gastrointestinales y episodios no significativos o no severos de hipoglucemia (13). El 36,6\% $(n=113)$ de nuestros pacientes se trataban con metformina. Se utilizó en una proporción similar a las sulfonilureas. El grupo tratado con metformina obtuvo valores más elevados de TG que los tratados con dieta e insulina, pero no hubo diferencias significativas entre grupos en relación al colesterol.

Los resultados de nuestro trabajo indican que numerosos FRC modificables en pacientes con DM 2 evaluados en nuestas consultas, no se encontraban dentro de los objetivos de buen control de acuerdo con las recomendaciones de la ADA.

Estudios realizados en pacientes diabéticos sugieren que existe una disminución del riesgo cardiovascular cuando se reduce la PAS $<130 \mathrm{mmHg}$ y la PAD $<85 \mathrm{mmHg}$ (8). En nuestro estudio el $36,2 \%$ de los pacientes presentó una TAD $<$ $80 \mathrm{mmHg}$ sin embargo solamente el 17,5\% alcanzaba los objetivos de control de la TAS $<130 \mathrm{mmHg}$. En estudios observacionales y epidemiológicos (16) se indica que en las personas diabéticas e hipertensas se incrementa hasta 4 veces el riesgo de un evento cardiovascular en comparación con poblaciones no diabéticas de la misma edad y sexo (9). Además, el riesgo de muerte cardiovascular aumenta en los diabéticos a medida que se incrementa la PAS (16), y una reducción de $10 \mathrm{mmHg}$ de la PAS en estos pacientes se asocia con una reducción del $12 \%$ de cualquier complicación relacionada con la DM, del $11 \%$ de infarto de miocardio y del $13 \%$ de complicaciones microvasculares (17). Debido a toda esta evidencia creemos que es recomendable seguir con el control agresivo de la HTA en pacientes diabéticos, tanto de la PAD, como de la PAS.

El 57,3\% de los paciente diabéticos de nuestro estudio no alcanzaban las cifras de $\mathrm{Hb} \mathrm{A} 1 \mathrm{C}<7 \%$ de buen control metabólico que recomienda la ADA. Sin embargo, se ha demostrado que un control glucémico agresivo en estos pacientes retrasa la aparición y la progresión de las complicaciones microvasculares de la diabetes, además de afectar favorablemente sobre un gran número de marcadores de enfermedad cardiovascular (3). En el UKPDS se ha demostrado que un control glucémico estricto se acompaña de una reducción del $16 \%$ en la morbilidad y mortalidad cardiovascular (3), por lo que es necesario el manejo agresivo de la glucemia en pacientes diabéticos. En nuestro estudio los pacientes en tratamiento con dieta tenían mejor control metabólico que los tratados con metformina e insulina (Tabla II). Esto podría deberse a un mejor estado metabólico con un menor deficit relativo de insulina, asociado a menos complicaciones y tiempo de evolución de la diabetes en estos pacientes, comparado con el resto de grupos de tratamiento. 
TABLA IV

PORCENTAJE DE PACIENTES QUE ALCANZAN OBJETIVOS DE CONTROL $\mathrm{n}=309$

\begin{tabular}{lcc}
\hline & $n$ & $\%$ \\
\hline IMC < 30 (Kg/ talla m $\left.{ }^{2}\right)$ & 154 & 49,8 \\
TAS < 130 (mmHg) & 53 & 17,1 \\
TAD < 80 (mmHg) & 114 & 36,9 \\
Colesterol < 200 (mg/dl) & 110 & 35,6 \\
Triglicéridos < 150 (mg/dl) & 156 & 50,5 \\
cHDL > 40 (mg/dl) & 174 & 56,3 \\
cLDL < 100 (mg/dl) & 50 & 16,2 \\
HbA1c < 7\% & 132 & 42,7 \\
Glucemia basal < 120 mg/dl & 60 & 19,4 \\
No fumar & 267 & 86,4 \\
\hline
\end{tabular}

IMC: indice de masa corporal. TAS: tensión arterial sistólica. TAD: tensión arterial diastólica. C-HDL: colesterol

unido a lipoproteínas de alta densidad. c-LDL: colesterol unido a lipoproteínasde baja densidad

Diversos estudios han demostrado una marcada disminución del riesgo cardiovascular al reducir los niveles de colesterol-LDL y aumentar los colesterol-HDL en pacientes diabéticos tratados con estatinas, recomendando reducir el colesterol- LDL a menos de $100 \mathrm{mg} / \mathrm{dl}$ en los mismos (2). En nuestro estudio a pesar de que el 43,7\% estaban en tratamiento con hipolipemiantes, solamente el $16,5 \%$ de los mismos alcanzó niveles de c-LDL menor de $100 \mathrm{mg} / \mathrm{dl}$. El 50,2\% alcanzó niveles de TG $<150$. Numerosos estudios han demostrado que la hipertrigliceridemia es un FRC independiente de las poblaciones diabéticas y que la reducción de los niveles de TG va a conseguir un descenso significativo de los episodios de cardiopatía isquémica (CI) en estos pacientes (2). Por ello, se aboga por un control estricto de los niveles de TG en pacientes diabéticos con CI.

La mayoría de estudios prospectivos han demostrado que el consumo de tabaco es un factor de riesgo independiente y modificable para la DM tipo 2 (4), y se asocia fuertemente a un incremento de riesgo de CI en pacientes diabéticos (18). Su abandono se acompaña sustancialmente de una disminución del riesgo de los mismos (18). En nuestro estudio el 13,6\% de nuestros pacientes eran fumadores (Tabla I), lo que coincide con otros estudios (16).

Se define como categoría de alto riesgo, el riesgo absoluto de desarrollar cardiopatía coronaria en el plazo de 10 años superior al 20\% (19). Existe actualmente un consenso generalizado de que los pacientes con DM tipo 2 deben ser incluidos en la categoría de alto riesgo, debido a la elevada asociación de esta enfermedad con factores de riesgo aterogénicos y a la mayor incidencia de eventos coronarios observados en estos pacientes, similar a la de pacientes no diabéticos con CI (16). En nuestro estudio la incidencia de CI fue del $11,7 \%$. Todo paciente con DM tipo 2 sin evidencia de CI tendría un riesgo equivalente al de aquellos pacientes en prevención secundaria, lo que les haría susceptibles de intervenciones propias de esta prevención.

El grado de obesidad y la distribución del tejido adiposo son factores predisponentes en la aparición de DM tipo 2, así como factores predictivos en la aparición de enfermedad coronaria. La mayoría de estudios realizados han demostrado que la obesidad abdominal con un incremento de grasa visceral se correlaciona significativamente con un perfil metabólico alterado que incluye resistencia a la insulina, hiperinsulinemia, intolerancia a la glucosa, hipertrigliceridemia, disminución de c-HDL, y aumento de c-LDL, que van a incrementar el riesgo de enfermedad coronaria (3). Además, recientemente se ha comprobado con los datos del estudio Framingham durante 44 años, que el efecto del sobrepeso (IMC: 25-29,9) sobre el incremento de riesgo de ECV es similar al que produce la obesidad (19). El 50\% de los diabéticos a estudio tenían un IMC mayor de 30, aunque hay que tener en cuenta que en nuestro trabajo todos los pacientes seleccionados eran obesos o con sobrepeso.

En los pacientes diabéticos, tanto los IECAs, como los ARAII han demostrado retrasar la aparición y enlentecer la progresión de la nefropatía diabética (20), así como reducir la mortalidad de origen cardiovascular (21). En este trabajo el 40,8 y $15,6 \%$ de los pacientes, se encontraban en tratamiento con IECAs y/o ARA II respectivamente. El bajo número de pacientes tratados con betabloqueantes $(15,9 \%)$ podría ser, en parte, debido al efecto de enmascaramiento de los síntomas de

TABLA V

COMPARACIÓN DE OBJETIVOS DE CONTROL ALCANZADOS POR GRUPOS DE TRATAMIENTO

\begin{tabular}{lccccc}
\hline & $\begin{array}{c}\text { Dieta } \\
n=102\end{array}$ & $\begin{array}{c}\text { Metformina } \\
n=97\end{array}$ & $\begin{array}{c}\text { Otros ADOs } \\
n=71\end{array}$ & $\begin{array}{c}\text { Insulina } \\
n=39\end{array}$ & Significación \\
\hline IMC $<30\left(\mathrm{Kg} / \mathrm{talla} \mathrm{m}{ }^{2}\right)$ & $50 \%$ & $44,33 \%$ & $56,33 \%$ & $51,28 \%$ & $\mathrm{NS}$ \\
TAS $<130(\mathrm{mmHg})$ & $13,72 \%$ & $23,71 \%$ & $15,49 \%$ & $12,82 \%$ & $\mathrm{NS}$ \\
TAD $<80(\mathrm{mmHg})$ & $39,22 \%$ & $37,11 \%$ & $32,39 \%$ & $38,46 \%$ & $\mathrm{NS}$ \\
Colesterol $<200(\mathrm{mg} / \mathrm{dl})$ & $36,27 \%$ & $28,87 \%$ & $38,03 \%$ & $46,15 \%$ & $\mathrm{NS}$ \\
Triglicéridos $<150(\mathrm{mg} / \mathrm{dl})$ & $59,80 \%$ & $38,14 \%$ & $47,88 \%$ & $61,54 \%$ & $\mathrm{p}=0.043$ \\
$\mathrm{CHDL}>40(\mathrm{mg} / \mathrm{dl})$ & $56,86 \%$ & $56,70 \%$ & $54,93 \%$ & $56,41 \%$ & $\mathrm{NS}$ \\
$\mathrm{CLDL}<100(\mathrm{mg} / \mathrm{dl})$ & $15,69 \%$ & $15,46 \%$ & $15,49 \%$ & $20,51 \%$ & $\mathrm{NS}$ \\
$\mathrm{HbA} 1 \mathrm{c}<7 \%$ & $57,80 \%$ & $30,92 \%$ & $42,25 \%$ & $33,33 \%$ & $\mathrm{p}<0.001$ \\
Glucemia basal $<120(\mathrm{mg} / \mathrm{dl})$ & $27,45 \%$ & $10,30 \%$ & $18,30 \%$ & $23,07 \%$ & $\mathrm{p}=0.049$ \\
No fumar & $84,31 \%$ & $84,54 \%$ & $88,73 \%$ & $89,74 \%$ & $\mathrm{NS}$ \\
\hline
\end{tabular}

Otros ADOs incluye cualquier antidiabético oral excepto la metformina.

IMC: indice de masa corporal. TAS: tensión arterial sistólica. TAD: tensión arterial diastólica. c-HDL: colesterol unido a lipoproteínas de alta densidad. 
hipoglucemia que presentan estos fármacos. Actualmente con la utilización de betabloqueantes cardioselectivos ha disminuido la aparición de este efecto (2). Aunque solamente el $34,6 \%$ de los pacientes diabéticos eran tratados con antiagregantes, tanto el ácido acetilsalicilico como el clopidogrel han demostrado reducir el riesgo de enfermedad isquémica, infarto de miocardio y mortalidad vascular en pacientes, como los diabéticos con riesgo de sufrir eventos isquémicos (22).

El tratamiento de la diabetes puede requerir por si misma 2 ó 3 tipos de medicamentos diarios. El diagnóstico de dislipemia, HTA, CI en el mismo paciente, lo cual es frecuente, podría aumentar el número de medicamentos a 8 ó 9 diarios. Esta situación podría dificultar la adhesión al tratamiento.

\section{CONCLUSIONES}

La metformina se utilizó en una proporción similar a las sulfonilureas en DM tipo 2 con sobrepeso u obesidad.

Nuestros resultados demuestran un mejorable control de los numerosos FRC de la población diabética estudiada, y ponen de manifiesto la necesidad de un control más estricto de los mismos para alcanzar los objetivos de control recomendados actualmente.

El diagnóstico precoz y una adecuada atención de los FRC pueden prevenir o retardar la aparición de complicaciones. Esto implica un enfoque multidisciplinar con una adecuada coordinación entre niveles asistenciales. Única- mente así podremos minimizar las consecuencias de la DM y sus complicaciones.

La evaluación de los resultados en salud y el grado de control en los pacientes sujetos a esta intervención programada, es un objetivo de especial trascendencia sanitaria, ya que, facilita el conocimiento de las características de la morbilidad de la población atendida, las posibles medidas de intervención para mejorar la prestación del servicio, así como una más adecuada utilización y asignación de recursos.

Desde el momento del diagnóstico de DM tipo 2 se debe intervenir sobre los FRC modificables que el paciente tenga alterados de acuerdo con las recomendaciones aceptadas actualmente y realizar un seguimiento periódico de los mismos.

En los obesos con DM tipo 2 no debería iniciarse ningún tratamiento farmacológico sin haber insistido en las posibilidades de la dieta, ejercicio y de la educación diabetológica. Un objetivo seria alcanzar el peso recomendable. Para conseguirlo debemos priorizar una dieta hipocalórica y la realización de ejercicio físico, pactar los objetivos individualizados con el paciente y que sean alcanzables.

Para realizar este control, se deben aunar esfuerzos de los médicos de atención primaria, especialistas y pacientes diabéticos para combatir la enfermedad cardiovascular. Los programas de educación dirigidos a los pacientes deben utilizarse como una parte integral del cuidado de los mismos. La educación diabetológica no sólo incrementa los conocimientos de los pacientes sobre su enfermedad, sino que también motiva al paciente para realizar adecuadamente el tratamiento.

\section{Bibliografía}

1. Martín Muñoz MC, Albarrán Juan ME, Lumbreras Marín EM. Revisión sobre el dolor neuropático en el síndrome del pie diabético. An Med Interna 2004; 21 (Supl. 9): 450-5.

2. Haffner SM, Alexander CM, Cook TJ, Bocuzzi SJ, Musliner TA, Pedersen TR. Reduced coronary events in simvastatin treated patients with coronary heart disease and diabetes or impaired fasting glucose levels. Arch Intern Med 1999; 159: 2661-7.

3. United Kingdom Prospective Study (UKPDS 34): Effect of intensive blood-glucose with metformin on complications in overweight patients with type 2 diabetes. Lancet 1998; 352: 854-65.

4. Wann a methee SG, Shaper AG, Peray IJ. Smoking as a modificable risk factor for type 2 diabetes mellitus in middle-aged men. Diabetes Care 2001; 24: 1590-5.

5. Wilson PW, Kannel WB. Obesity, diabetes and risk of cardiovascular disease in the elderly. Am J Geriatr cardiol 2002; 11: 119-23.

6. Mundet Tuduri X, Carmena Jimenez F, Gussinyer Canabal P. Complicaciones crónicas de la diabetes mellitus tipo 2. Evolución tras 5 años de seguimiento. Aten Primaria 2000; 25: 405-11.

7. Gimeno Orna JA, Boned Juliani B, Lou Arnal M, Castro Alonso FJ. Factores relacionados con el control glucémico de pacientes con diabetes mellitus tipo 2. An Med Interna (Madrid) 2003; 20: 122-6.

8. Morrish NJ, Wang SL, Stevens LK, Fuller JH, Keen H and the WHO Multinational Study Group. Mortality and causes of death in the WHO multinational study of vascular disease in diabetes. Diabetología 2001, 44 (Supl. 2): 14-22.

9. Alder AL, Stratton IM, Neil HA, Yudkin JS, Matthews DR, Cull CA, Wright AD, Turner RC, Holmann RR. Association of systolic blood with macrovascular and microvascular complications in type 2 diabetes (UKPDS 36). Prospective observational study. BMJ 2000; 321: 412-9.

10. European Diabetes policy Group. A desktop guide to type 2 diabetes mellitus. Diabet Med 1999; 16: 716-30.

11. Standards of medical care with patients with diabetes mellitus. American Diabetes Asociation. Diabetes Care 2004; 27 (Supl.1).

12. Aviles-Santa L, Sinding J, Raskin P. Effects of metformin in patients with poorly controlled, insulin treated type 2 diabetes mellitus. Ann Intern Med 1999; 131: 182-8.

13. Jaber A, Pharm D, Nowak N, Slaughter R. Insulin-Metformin Combination Therapy in Obese Patients with Type 2 Diabetes. J Clin Pharmacol 2002; 42: 89-94

14. Wiernsperger NF, Bailey CJ. The antihyperglycaemic effect of metformin: therapeutic and cellular mechanisms. Drugs 1999; 58 (Supl. 1): 31-39.

15. United Kingdom Prospective Stydy (UKPDS) Group: Glycemic control with diet, sulfonylurea, metformin, or insulin in patients with type 2 diabetes mellitus, progressive requirements for multiple therapies (UKPDS 49). JAMA 1999; 281: 2005-12.

16. George PB, Tobin KJ, Corpus RA, Devlin WH, O`Neill WW. Treatment of cardiac risk factors in diabetic patients: how well do we follow the Guidelines?. Am Heart J 2001; 142: 857-63.

17. Tuomiletho J, Rastenyle D, Birkenhager WH, Thijs L, AntiKainen R, Bulpitt CJ, Fletcher AE, Forette F, Goldhaber A, Palatini P, Sarti C, Fagaro R. Effects of calcium channel blockade in older patients with diabetes and systolic hypertension. N Engl J Med 1999; 340: 677-84.

18. Al-Delaimy WK, Manson JE, Solomon CG, Kanachi I, Stampfer MJ, Willett WC, Hu FB. Smoking andrisk of coronary heart disease amony women with type 2 diabetes mellitus. Arch Intern Med 2003; 162: 273-9.

19. Wilson PW, Dágostino RB, Sullivan L, Parise H, Kannel WB. Overweight and obesity as determinants of cardiovascular risk: the Framingham Experience. Arch Intern Med 2002; 162: 1867-72.

20. Parving HH, Lehnert H, Bröchner-Mortensen J, Gomis R, Andersen S, Arner P. The effect of irbesartan on the development of diabetic nephropaty in patients with type 2 diabetes. N Engl J Med 2001; 345: 870-8.

21. Lewis EJ, Hunsicker LG, Clarke WR, Berl T, Pohl MA, Lewis JB, Ritz E, Atkins RC, Rohde R, Raz I. Renoprotective effect of the angiotensin receptor antaogonist irbesartan in patients with nephropathy due to type 2 diabetes. N Engl J Med 2001; 345: 851-60.

22. The CAPRIL Steering Comitte. A randomized blinded trial of clopidogrel versus aspirin in patients as risk of ischaemic events. Lancet 1998; 398: 1329-39. 